\title{
Review of recent theoretical developments in Higgs physics
}

\section{Radja Boughezal ${ }^{* i}$}

High Energy Physics Division, Argonne National Laboratory, Argonne, IL 60439, US

E-mail: rboughezaleanl.gov

We briefly review the current status of theoretical predictions for Higgs production at hadron colliders within the Standard Model. We summarize the main production mechanisms at the LHC and focus on issues related to the gluon fusion mode.

Xth Quark Confinement and the Hadron Spectrum,

October 8-12, 2012

TUM Campus Garching, Munich, Germany

\footnotetext{
* Speaker.

${ }^{\dagger}$ We thank the organizers for the kind invitation to this conference. This work is supported by the U.S. Department of Energy, Division of High Energy Physics, under contract DE-AC02-06CH11357.
} 


\section{Introduction}

The recent discovery of a new boson by the ATLAS and CMS collaborations at the LHC $[1,2]$ has ushered in a new era in particle physics. The future program of the LHC, and the next stage of experimental studies in high energy physics, will be largely devoted to measuring and understanding the properties of the new state in order to determine the underlying theory from which it arises. The initial data provides only a hazy glimpse at the properties of the new particle. Observation of its decay into two photons indicates that it cannot be a spin-one state, according to the Landau-Yang theorem [3]. Initial measurements of its branching ratios into various final states indicates that its couplings are consistent with those predicted for the Standard-Model Higgs boson, as determined by the experimental collaborations and by several independent analyses $[4,5,6]$. The slight excess observed over Standard Model predictions in the $\gamma \gamma$ final state has already received explanations both within [7] and beyond the Standard Model [8, 9, 10, 11, 12, 13, 14, 15, 16, 17]. While the recent discovery has strongly constrained the parameter space of several models [18, 19, 20, 21], significant work will clearly be needed to sharpen our picture of the new state. Searches for additional " top-quark partners" at the LHC will be needed to test whether the discovered boson obeys the principle of naturalness [22, 23, 24, 25]

The Standard Model Higgs coupling is strongest to the heaviest particles. Therefore, we distinguish three types of decays: into fermions, into massive gauge bosons and loop-induced decays through a massive loop of quarks or gauge bosons. The LHC may be able to provide a measurement of the Higgs coupling at the $10-30 \%$ level [26, 27]. Precise theoretical predictions for these decays are therefore needed. Backgrounds to the Higgs signal are severe in many channels, particularly when a mass peak cannot be reconstructed such as in $H \rightarrow W W \rightarrow l v l v$, and knowledge of the signal shape and normalization is needed to optimize experimental searches. Signal and background cross sections must therefore be predicted as accurately as can be achieved.

Higgs production at both hadron colliders, Tevatron and LHC, is dominated by gluon fusion, where two incoming gluons produce a Higgs boson via a virtual top quark loop. This is followed by vector boson fusion (VBF), where the incoming protons radiate a $\mathrm{W}$ or a $\mathrm{Z}$ boson, which subsequently interact weakly and fuse into a Higgs boson. The Higgs can also be produced in association with a pair of top quarks or through Higgs strahlung (associated WH or ZH production).

In this short review, we summarize the status of theoretical predictions for Higgs signal and background processes at hadron colliders. In addition, we discuss issues related to the effect of jet vetos on estimating the uncertainties in the gluon fusion cross section, resummation of the jet veto induced large logarithms, as well as recent proposed ideas for analyzing the Higgs properties. More details about some of these issues can be found in [28, 29, 30]

\section{Gluon Fusion}

The dominant production mode of the Standard Model Higgs boson at the Tevatron and LHC is gluon fusion, mediated by a heavy-quark loop, with a cross section that is a factor of 10 larger than all other production modes cross sections (see Fig. (1)). Radiative QCD corrections to this 


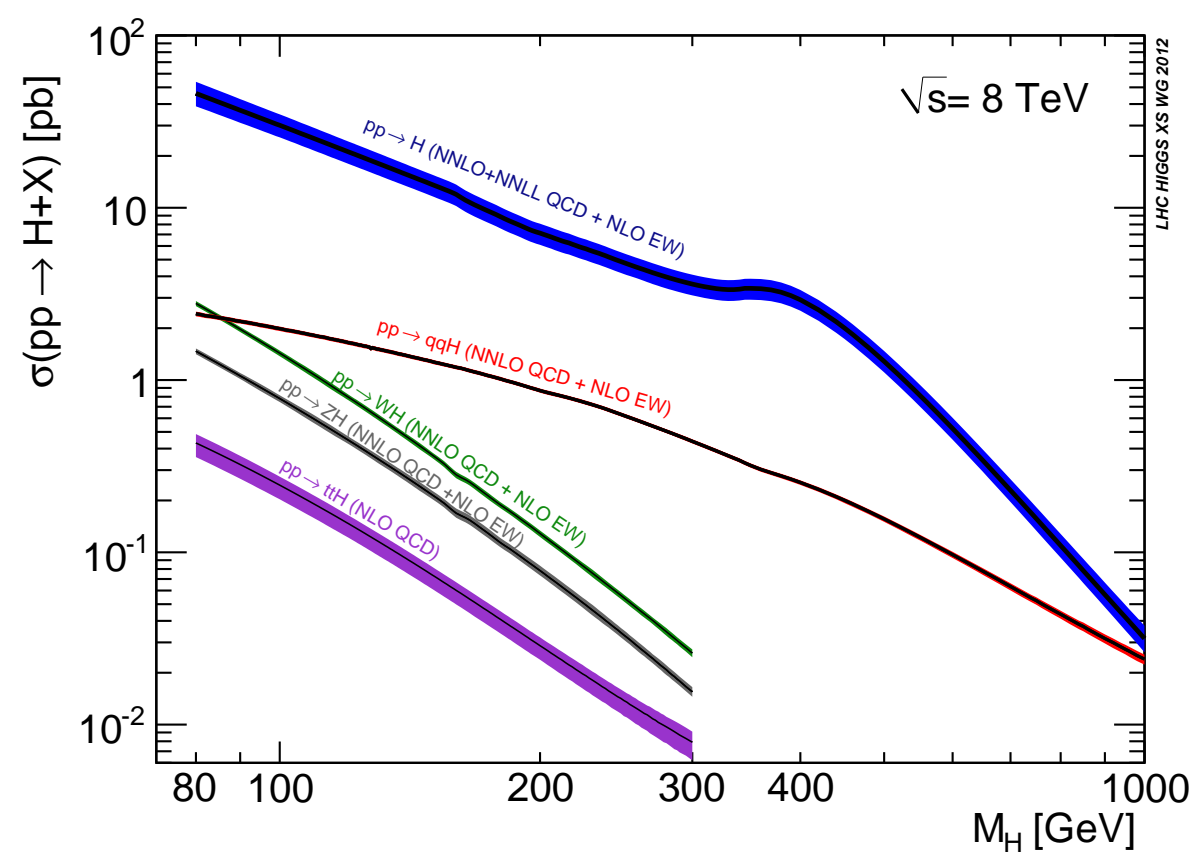

Figure 1: Total cross sections for Higgs production at the LHC at $\sqrt{s}=8 \mathrm{TeV}$.

process turned out to be very important. At the NLO level, they were found to increase the LO cross section by about $80-100 \%[31,32,33]$. The gluon-Higgs interaction seems to be very well approximated by an effective Lagrangian obtained by decoupling the top quark [34]

$$
\mathscr{L}_{e f f}=-\alpha_{s} \frac{C_{1}}{4 \mathrm{v}} H G_{\mu \nu}^{a} G^{a \mu \nu},
$$

if the exact Born cross section with the full dependence on the top and bottom quark masses is used to normalize the result. The difference between the exact and the approximate NLO cross sections is less than $1 \%$ for Higgs masses up to $200 \mathrm{GeV}$, and does not exceed $10 \%$ even for Higgs masses up to $1 \mathrm{TeV}$, well far away from its formal range of validity $M_{H}<2 M_{t o p}$. In equation (2.1), $\mathrm{v}$ is the vacuum expectation value of the Higgs field, $\mathrm{v}=246 \mathrm{GeV}$, and $C_{1}$ is the Wilson coefficient, currently known through $\alpha_{s}^{5}[35,36]$. In this large $M_{\text {top }}$ limit, the NNLO QCD corrections were computed in [37, 38, 39], leading to an additional increase of the cross section of roughly $10-15 \%$, and showing a good convergence of the perturbative series. Very recently, the three-loop virtual corrections to this process, where finite top quark mass effects are taken into account, were presented in [40, 41].

A further increase in the cross section of about $6 \%$ was obtained by doing soft-gluon resummation [42]. This result was confirmed through the leading soft contributions at $N^{3} L O$ [43, 44, 45]. Taking all the perturbative effects into account, the inclusive result of the cross section increases by a factor of 2 at LHC and 3.5 at Tevatron. The theoretical uncertainty from effects beyond NNLO was estimated to be about $\pm 10 \%$ by varying the renormalization and factorization scales.

The largeness of the K-factors at both colliders is an open question. In [46], the authors argue 
that this is due to enhanced contributions of the form $\left(C_{A} \pi \alpha_{s}\right)^{L}$, coming from the analytic continuation of the gluon form factor to time-like momentum transfer, with $L$ being the number of loops. A resummation of these terms using soft collinear effective theory (SCET) leads to smaller values of the K-factors; in fact at the LHC, the K-factor for small values of $M_{H}$ is close to 1.

The importance and success in taming the QCD corrections to Higgs production have shifted attention to electroweak corrections to the Higgs signal. The authors of Refs. [47, 48] pointed out important 2-loop light-quark effects; the corresponding diagrams involve the Higgs coupling to $W$ or Z-bosons which then couple to gluons through a light-quark loop. These terms are not suppressed by light-quark Yukawa couplings, and receive a multiplicity enhancement from summing over the quarks. A careful study of the full 2-loop electroweak effects was performed in Ref. [49]. They increase the leading-order cross section by up to $5-6 \%$ for relevant Higgs masses. The leading order of the mixed QCD-electroweak corrections, due to diagrams containing light quarks, was calculated in [50], using an effective field theory approach where the $\mathrm{W}$ boson is integrated out. This work allowed to check the complete factorization hypothesis of QCD and electroweak corrections proposed in Ref. [48, 49]. The result shows that, despite the large violation of the factorization assumption, a significant numerical difference from the prediction of this hypothesis is not observed in the cross section, due to the dominant QCD corrections. The end effect on the cross section is an additional enhancement of up to $6 \%$ from the $\mathscr{O}(\alpha)+\mathscr{O}\left(\alpha \alpha_{s}\right)$ terms.

In addition to the previous results, the authors of [50] provided a new prediction for the inclusive cross section of the gluon fusion process. This result takes into account all the new theoretical calculations: the 2-loop light-quark diagrams based on the complex-mass scheme for the $W$-and $Z$-bosons [49], the new 3-loop $\mathscr{O}\left(\alpha \alpha_{s}\right)$ correction, the contributions from top and bottom quarks with the exact NLO K-factors and at the time the newest parton distribution functions (PDF) by the MSTW group [51, 52,53]. These same effects were later on also taken into account in a prediction provided by the authors of $[54,55]$. Both groups calculate the uncertainty on the cross section due to missing higher order corrections by varying the renormalization and factorization scales in the range $\mu_{0} / 2<\mu_{F}, \mu_{R}<2 \mu_{0}$, with $\mu_{0}=m_{H} / 2$ for the first group and $\mu_{0}=m_{H}$ for the second one. They obtain similar values for the uncertainty, roughly $8-10 \%$. Other predictions for the gluon fusion inclusive cross section were provided by two other groups with values of the uncertainty on the cross section that have been debated in the community [56, 57, 58]. For more details we refer the reader to [28].

The calculations mentioned above refer to the inclusive cross section, which means no experimental cuts were imposed. It was shown in a previous work [59] that the impact of higher order corrections on the rate and shape of the corresponding distributions may be strongly dependent on the chosen cuts. The most general higher order prediction for gluon fusion process is available in the form of partonic NNLO Monte Carlo programs $[60,61]$ which use the large- $M_{\text {top }}$ limit and vanishing b-quark mass. In [59], the authors have shown that, while for the process $g g \rightarrow H \rightarrow \gamma \gamma$, radiative corrections are only slightly affected by the signal cuts, the process $g g \rightarrow H \rightarrow W W \rightarrow l v \bar{l} v$ is strongly affected by these cuts which take away most of the increase observed in the inclusive cross section. Additionaly, finite top- and bottom-quark mass effects and electroweak contributions 
to the Higgs boson transverse momentum spectrum $\left(P_{T}\right.$-spectrum) at the NLO level were presented in $[62,63,64]$. In a recent work, the authors of [65] have combined the results of the NNLO QCD corrections to the inclusive cross section with the resummation of multiple soft gluon emissions at small transverse momenta at the next-to-next-to-leading logarithmic (NNLL) accuracy, in a numerical program HRes, which is publicly available.

In searching for the Higgs at hadron colliders, it is customary to divide the data into exclusive jet bins in order to reduce the background. Traditionally, the uncertainty on the exclusive fixed order predictions was estimated by varying the renormalization and factorization scales, similarly to the inclusive case. The authors of [66] have shown that this way of calculating the perturbative uncertainty underestimates it due to cancellations between the perturbative corrections. They have proposed a way of evaluating the uncertainty separately for each jet multiplicity then combining them to get the final result taking into account the correlation between the uncertainties in the different jet bins as well as in the total cross section. The jet veto uncertainties in Higgs production was also discussed by the authors of [67], who have in addition studied the jet veto efficiency and have done a next-to-leading logarithmic (NLL) resummation in the transverse momentum threshold used for identifying jets. Attempts for extending this resumation to the NNLL approximation have then followed by three groups $[68,69,70]$ and to the Higgs +1 jet process at NLL in [71] .

\subsection{Vector Boson Fusion}

This process is important for the discovery of the Higgs boson at the LHC for a wide range of Higgs masses [75, 76, 77, 78]. The vector boson fusion (VBF) cross section is one order of magnitude lower than the one for gluon fusion, but it is an attractive channel for measurements of the Higgs couplings and CP properties [72, 73, 26, 74]. In VBF, the Higgs is produced in association with two jets which are scattered into the forward direction. These two jets are not color connected at LO, which means that the hadronic activity in the rapidity region between these two jets is very small. On the other hand, the Higgs decay products are found at central rapidities, which allows to efficiently reduce the background if suitable cuts are chosen.

The NLO QCD corrections to the total cross section were computed a long time ago and found to be of the order 5-10\% [79]. These corrections have been implemented in fully differential partonic NLO Monte Carlo programs in [81, 84]. Approximate NNLO QCD results using the structure function approach are also known [83] leading to an overall theoretical uncertainty of $1-2 \%$ The full electroweak and QCD corrections to this process have also been computed [82, 84]. Like other production modes, the VBF process suffers from a large background. The dominant one when trying to isolate the $H W W$ and $H Z Z$ couplings through VBF is the Higgs production plus two jets from gluon-gluon fusion. The LO contribution to this background is known keeping the full top-mass dependence [85]. The authors have shown that the VBF cuts on the rapidity and the transverse momentum of the tagging jets work efficiently in this case. The NLO QCD corrections to $H j j$ in the large top quark mass limit are also known [86], as well as parton shower effects on the azimuthal angle correlation of the two jets and the rapidity distribution of extra jets [87].

\subsection{Higgs Strahlung}

This is the third important channel at the LHC after gluon fusion and vector boson fusion, 
based on the inclusive cross section. Although it was considered less promising due to the large background, a recent analysis has shown that a signal in this channel might be observable [88], by looking at events where both the Higgs and the gauge boson $\left(W^{ \pm}\right.$or Z) have large transverse momentum. The NLO QCD corrections to this production mode are known [89]. They increase the cross section by about 30\%. The NNLO QCD corrections result from two subsets of diagrams: a Drell-Yan production of a vector boson that radiates a Higgs boson, and diagrams where the Higgs is produced through a heavy quark loop. Results for the first set of diagrams were available several years ago and give a further increase the cross section by about $5-10 \%$ [90]. These corrections lead to a reduction of the scale dependence of the cross section from $10 \%$ at LO to $5 \%$ at NLO, to $2 \%$ when the NNLO result is included. The second subset of diagrams was evaluated recently [91], their effect is of the order $1-3 \%$. At this level of precision, electroweak corrections become important to further improve the precision of the prediction. They were calculated at order $\mathscr{O}(\alpha)$ in [92] and were found to decrease the cross section by $5 \%$ to $10 \%$ depending on the Higgs boson mass and the input parameters scheme. A fully exclusive NNLO QCD calculation for the WH production mode was recently presented in [93], allowing arbitrary cuts on the $\mathrm{W}$ and Higgs decay products. By searching for events where the Higgs is boosted at high $P_{T}$ at the LHC, sizable QCD corrections were found, thereby challenging the stability of the fixed-order calculation.

\section{Methods for analysing Higgs properties}

Two of the first quantities requiring determination for the recently discovered boson by the LHC collaborations are the spin and CP properties. These can be measured through a variety of ways in multiple final states. An initial attempt to determine the CP properties assuming a spin-zero state has been made [94], and a discussion of how to disentangle the various spin and CP possibilities once more data is taken was presented in Ref. [95]. As the largest experimental excesses are in the $\gamma \gamma$ and $Z Z \rightarrow 4 l$ final states, where either one or both $Z$-bosons are off-shell, initial studies will focus on these two modes. Two broad categories of techniques exist for the measurement of the new particle's quantum numbers. Multi-variate methods input all of the kinematic information in an event into a likelihood function that can be used to exclude hypotheses for the new state's identity. These ideas have been suggested in the literature for both analyzing the new particle's properties and assisting in discovery [96, 97, 98], and in particular are heavily used in the CMS analysis of the ZZ final state [2]. In the large-time and large-data set limit, such techniques provide the most sensitivity to particle properties, since no kinematic information is neglected. Alternatively, single variables that provide sensitivity to properties of interest can be studied. Analyses of this type offer the advantages of simplicity and clarity over multi-variate approaches, and can be more easily implemented to provide answers quickly. They can also indicate which input variables should be used to improve the efficiency of multi-variate techniques.

In [99], the authors have introduced a powerful single-variable measurement in the four-lepton final state that discriminates among both the spin and CP possibilities for the new state. This method can be used when either one or both intermediate $Z$ bosons are off-shell, and is relatively insensitive to background contamination. The idea is simple to explain. Let $M_{12}$ and $M_{34}$ respectively denote the same-flavor lepton pairs with the highest and lowest invariant masses. We can suggestively call the mass of the heavy resonance decaying to the leptons $M_{H} . M_{34}$ must satisfy the 
following inequality: $M_{34} \leq M_{H}-M_{12}$. The fall-off of the $M_{34}$ distribution as this upper limit is approached is sensitive to the spin and CP nature of the heavy resonance. Denoting the momentum of the 34-pair in the $H$ rest frame as $\beta$, for a pure CP-even spin-zero state this distribution decreases linearly in $\beta$. For a pure CP-odd spin-zero state, it falls off as $\beta^{3}$. For spin-two states, it falls off as either $\beta, \beta^{3}$, or $\beta^{5}$, depending on the couplings of this state to spin-one particles. Measurement of this distribution provides a powerful handle on the couplings of this new state. More details about this method can be found in [99].

\section{Summary}

We have briefely reviewed the current theoretical status of the most important production modes of a Standard Model Higgs boson at hadron colliders. The recent discovery of a new boson by the ATLAS and CMS collaborations at the LHC has ushered in a new era in particle physics. The next stage of experimental and theoretical studies in high energy physics, will be to understand the properties of the new state in order to determine the underlying theory from which it arises.

\section{References}

[1] G. Aad et al. [ATLAS Collaboration], [arXiv:1207.7214 [hep-ex]].

[2] S. Chatrchyan et al. [CMS Collaboration], [arXiv:1207.7235 [hep-ex]].

[3] L. D. Landau, Dokl. Akad. Nawk., USSR 60, 207 (1948); C. N. Yang, Phys. Rev. 77, 242 (1950).

[4] D. Carmi, A. Falkowski, E. Kuflik, T. Volansky and J. Zupan, arXiv:1207.1718 [hep-ph].

[5] T. Plehn and M. Rauch, arXiv:1207.6108 [hep-ph].

[6] A. Djouadi, arXiv:1208.3436 [hep-ph].

[7] J. Baglio, A. Djouadi and R. M. Godbole, arXiv:1207.1451 [hep-ph].

[8] A. Joglekar, P. Schwaller and C. E. M. Wagner, arXiv:1207.4235 [hep-ph].

[9] G. F. Giudice, P. Paradisi and A. Strumia, arXiv:1207.6393 [hep-ph].

[10] A. Delgado, G. Nardini and M. Quiros, arXiv:1207.6596 [hep-ph].

[11] M. Hashimoto and V. A. Miransky, arXiv:1208.1305 [hep-ph].

[12] K. Howe and P. Saraswat, arXiv:1208.1542 [hep-ph].

[13] T. Li, J. A. Maxin, D. V. Nanopoulos and J. W. Walker, arXiv:1208.1999 [hep-ph].

[14] R. Sato, K. Tobioka and N. Yokozaki, arXiv:1208.2630 [hep-ph].

[15] Z. Kang, T. Li, J. Li and Y. Liu, arXiv:1208.2673 [hep-ph].

[16] K. J. Bae, K. Choi, E. J. Chun, S. H. Im, C. B. Park and C. S. Shin, arXiv:1208.2555 [hep-ph].

[17] H. Davoudiasl, H. -S. Lee and W. J. Marciano, arXiv:1208.2973 [hep-ph].

[18] R. Boughezal and F. Petriello, Phys. Rev. D 81, 114033 (2010) [arXiv:1003.2046 [hep-ph]].

[19] R. Boughezal, Phys. Rev. D 83, 093003 (2011) [arXiv:1101.3769 [hep-ph]].

[20] C. Anastasiou, R. Boughezal and E. Furlan, JHEP 1006, 101 (2010) [arXiv:1003.4677 [hep-ph]]. 
[21] E. Kuflik, Y. Nir and T. Volansky, arXiv:1204.1975 [hep-ph].

[22] S. Dimopoulos and H. Georgi, Nucl. Phys. B 193, 150 (1981).

[23] H. -C. Cheng and I. Low, JHEP 0309, 051 (2003); JHEP 0408, 061 (2004).

[24] C. Brust, A. Katz, S. Lawrence and R. Sundrum, JHEP 1203, 103 (2012); M. Papucci, J. T. Ruderman and A. Weiler, JHEP 1209, 035 (2012).

[25] R. Boughezal and M. Schulze, arXiv:1212.0898 [hep-ph].

[26] M. Duhrssen, S. Heinemeyer, H. Logan, D. Rainwater, G. Weiglein and D. Zeppenfeld, Phys. Rev. D 70 (2004) 113009 [arXiv:hep-ph/0406323].

[27] R. Lafaye, T. Plehn, M. Rauch, D. Zerwas and M. Duhrssen, arXiv:0904.3866 [hep-ph].

[28] S. Dittmaier et al. [LHC Higgs Cross Section Working Group Collaboration], arXiv:1101.0593 [hep-ph].

[29] S. Dittmaier, S. Dittmaier, C. Mariotti, G. Passarino, R. Tanaka, S. Alekhin, J. Alwall and E. A. Bagnaschi et al., arXiv:1201.3084 [hep-ph].

[30] R. Boughezal, arXiv:0908.3641 [hep-ph].

[31] S. Dawson, Nucl. Phys. B 359, 283 (1991).

[32] A. Djouadi, M. Spira and P. M. Zerwas, Phys. Lett. B 264, 440 (1991).

[33] D. Graudenz, M. Spira and P. M. Zerwas, Phys. Rev. Lett. 70, 1372 (1993); M. Spira, A. Djouadi, D. Graudenz and P. M. Zerwas, Nucl. Phys. B 453, 17 (1995) [arXiv:hep-ph/9504378].

[34] M. 1. Kramer, E. Laenen and M. Spira, Nucl. Phys. B 511 (1998) 523 [arXiv:hep-ph/9611272].

[35] Y. Schroder and M. Steinhauser, JHEP 0601 (2006) 051 [arXiv:hep-ph/0512058].

[36] K. G. Chetyrkin, J. H. Kuhn and C. Sturm, Nucl. Phys. B 744 (2006) 121 [arXiv:hep-ph/0512060].

[37] R. V. Harlander and W. B. Kilgore, Phys. Rev. Lett. 88, 201801 (2002) [arXiv:hep-ph/0201206].

[38] C. Anastasiou and K. Melnikov, Nucl. Phys. B 646, 220 (2002) [arXiv:hep-ph/0207004].

[39] V. Ravindran, J. Smith and W. L. van Neerven, Nucl. Phys. B 665, 325 (2003) [arXiv:hep-ph/0302135].

[40] R. V. Harlander and K. J. Ozeren, arXiv:0907.2997 [hep-ph].

[41] A. Pak, M. Rogal and M. Steinhauser, arXiv:0907.2998 [hep-ph].

[42] S. Catani, D. de Florian, M. Grazzini and P. Nason, JHEP 0307 (2003) 028 [arXiv:hep-ph/0306211].

[43] S. Moch and A. Vogt, Phys. Lett. B 631 (2005) 48 [arXiv:hep-ph/0508265].

[44] E. Laenen and L. Magnea, Phys. Lett. B 632 (2006) 270 [arXiv:hep-ph/0508284].

[45] A. Idilbi, X. d. Ji, J. P. Ma and F. Yuan, Phys. Rev. D 73 (2006) 077501 [arXiv:hep-ph/0509294].

[46] V. Ahrens, T. Becher, M. Neubert and L. L. Yang, Eur. Phys. J. C 62 (2009) 333 [arXiv:0809.4283 [hep-ph]].

[47] U. Aglietti, R. Bonciani, G. Degrassi and A. Vicini, Phys. Lett. B 595, 432 (2004) [arXiv:hep-ph/0404071].

[48] U. Aglietti, R. Bonciani, G. Degrassi and A. Vicini, arXiv:hep-ph/0610033. 
[49] S. Actis, G. Passarino, C. Sturm and S. Uccirati, arXiv:0809.1301 [hep-ph]; S. Actis, G. Passarino, C. Sturm and S. Uccirati, arXiv:0809.3667 [hep-ph].

[50] C. Anastasiou, R. Boughezal and F. Petriello, JHEP 0904 (2009) 003 [arXiv:0811.3458 [hep-ph]].

[51] A. D. Martin, W. J. Stirling, R. S. Thorne and G. Watt, arXiv:0901.0002 [hep-ph]

[52] A. D. Martin, W. J. Stirling, R. S. Thorne and G. Watt, arXiv:0905.3531 [hep-ph]

[53] R. S. Thorne, A. D. Martin, W. J. Stirling and G. Watt, arXiv:0907.2387 [hep-ph].

[54] D. de Florian and M. Grazzini, Phys. Lett. B 674, 291 (2009) [arXiv:0901.2427 [hep-ph]].

[55] D. de Florian and M. Grazzini, Phys. Lett. B 718, 117 (2012) [arXiv:1206.4133 [hep-ph]].

[56] J. Baglio and A. Djouadi, JHEP 1010, 064 (2010) [arXiv:1003.4266 [hep-ph], arXiv:1009.1363 [hep-ph]].

[57] J. Baglio and A. Djouadi, JHEP 1103, 055 (2011) [arXiv:1012.0530 [hep-ph]].

[58] V. Ahrens, T. Becher, M. Neubert and L. L. Yang, Phys. Lett. B 698, 271 (2011) [arXiv:1008.3162 [hep-ph]].

[59] C. Anastasiou, G. Dissertori and F. Stockli, JHEP 0709 (2007) 018 [arXiv:0707.2373 [hep-ph]].

[60] C. Anastasiou, K. Melnikov and F. Petriello, Nucl. Phys. B 724 (2005) 197 [arXiv:hep-ph/0501130].

[61] S. Catani and M. Grazzini, Phys. Rev. Lett. 98 (2007) 222002 [arXiv:hep-ph/0703012].

[62] W. Y. Keung and F. J. Petriello, Phys. Rev. D 80 (2009) 013007 [arXiv:0905.2775 [hep-ph]].

[63] C. Anastasiou, S. Bucherer and Z. Kunszt, arXiv:0907.2362 [hep-ph].

[64] O. Brein, Phys. Rev. D 81, 093006 (2010) [arXiv:1003.4438 [hep-ph]].

[65] D. de Florian, G. Ferrera, M. Grazzini and D. Tommasini, JHEP 1206, 132 (2012) [arXiv:1203.6321 [hep-ph]].

[66] I. W. Stewart and F. J. Tackmann, Phys. Rev. D 85, 034011 (2012) [arXiv:1107.2117 [hep-ph]].

[67] A. Banfi, G. P. Salam and G. Zanderighi, JHEP 1206, 159 (2012) [arXiv:1203.5773 [hep-ph]].

[68] A. Banfi, P. F. Monni, G. P. Salam and G. Zanderighi, Phys. Rev. Lett. 109, 202001 (2012) [arXiv:1206.4998 [hep-ph]].

[69] F. J. Tackmann, J. R. Walsh and S. Zuberi, Phys. Rev. D 86, 053011 (2012) [arXiv:1206.4312 [hep-ph]].

[70] T. Becher and M. Neubert, JHEP 1207, 108 (2012) [arXiv:1205.3806 [hep-ph]].

[71] X. Liu and F. Petriello, arXiv:1210.1906 [hep-ph].

[72] D. Zeppenfeld, R. Kinnunen, A. Nikitenko and E. Richter-Was, Phys. Rev. D 62 (2000) 013009 [arXiv:hep-ph/0002036].

[73] A. Belyaev and L. Reina, JHEP 0208 (2002) 041 [arXiv:hep-ph/0205270].

[74] G. Klamke and D. Zeppenfeld, JHEP 0704 (2007) 052 [arXiv:hep-ph/0703202].

[75] D. L. Rainwater and D. Zeppenfeld, JHEP 9712 (1997) 005 [arXiv:hep-ph/9712271].

[76] D. L. Rainwater, D. Zeppenfeld and K. Hagiwara, Phys. Rev. D 59 (1999) 014037 [arXiv:hep-ph/9808468]. 
[77] T. Plehn, D. L. Rainwater and D. Zeppenfeld, Phys. Rev. D 61 (2000) 093005 [arXiv:hep-ph/9911385].

[78] O. J. P. Eboli and D. Zeppenfeld, Phys. Lett. B 495 (2000) 147 [arXiv:hep-ph/0009158].

[79] T. Han, G. Valencia and S. Willenbrock, Phys. Rev. Lett. 69 (1992) 3274 [arXiv:hep-ph/9206246].

[80] S. Asai et al., Eur. Phys. J. C 32S2 (2004) 19 [arXiv:hep-ph/0402254].

[81] K. Arnold, M. Bahr, G. Bozzi, F. Campanario, C. Englert, T. Figy, N. Greiner and C. Hackstein et al., Comput. Phys. Commun. 180, 1661 (2009) [arXiv:0811.4559 [hep-ph]].

[82] M. Ciccolini, A. Denner and S. Dittmaier, Phys. Rev. Lett. 99 (2007) 161803 [arXiv:0707.0381 [hep-ph]].

[83] P. Bolzoni, M. Zaro, F. Maltoni and S. -O. Moch, Nucl. Phys. Proc. Suppl. 205-206, 314 (2010) [arXiv:1006.2323 [hep-ph]].

[84] M. Ciccolini, A. Denner and S. Dittmaier, Phys. Rev. D 77 (2008) 013002 [arXiv:0710.4749 [hep-ph]].

[85] V. Del Duca, W. Kilgore, C. Oleari, C. Schmidt and D. Zeppenfeld, Nucl. Phys. B 616 (2001) 367 [arXiv:hep-ph/0108030].

[86] J. M. Campbell, R. K. Ellis and G. Zanderighi, JHEP 0610 (2006) 028 [arXiv:hep-ph/0608194].

[87] V. Del Duca et al., JHEP 0610 (2006) 016 [arXiv:hep-ph/0608158].

[88] J. M. Butterworth, A. R. Davison, M. Rubin and G. P. Salam, Phys. Rev. Lett. 100 (2008) 242001 [arXiv:0802.2470 [hep-ph]].

[89] T. Han and S. Willenbrock, Phys. Lett. B 273 (1991) 167.

[90] O. Brein, A. Djouadi and R. Harlander, Phys. Lett. B 579, 149 (2004) [arXiv:hep-ph/0307206].

[91] O. Brein, R. Harlander, M. Wiesemann and T. Zirke, Eur. Phys. J. C 72, 1868 (2012) [arXiv:1111.0761 [hep-ph]].

[92] M. L. Ciccolini, S. Dittmaier and M. Kramer, Phys. Rev. D 68 (2003) 073003 [arXiv:hep-ph/0306234].

[93] G. Ferrera, M. Grazzini and F. Tramontano, Phys. Rev. Lett. 107, 152003 (2011) [arXiv:1107.1164 [hep-ph]].

[94] B. Coleppa, K. Kumar and H. E. Logan, arXiv:1208.2692 [hep-ph].

[95] S. Bolognesi, Y. Gao, A. V. Gritsan, K. Melnikov, M. Schulze, N. V. Tran, and A. Whitbeck, arXiv:1208.4018 [hep-ph].

[96] Y. Gao, A. V. Gritsan, Z. Guo, K. Melnikov, M. Schulze and N. V. Tran, Phys. Rev. D 81, 075022 (2010) [arXiv:1001.3396 [hep-ph]].

[97] A. De Rujula, J. Lykken, M. Pierini, C. Rogan and M. Spiropulu, Phys. Rev. D 82, 013003 (2010) [arXiv:1001.5300 [hep-ph]].

[98] J. S. Gainer, K. Kumar, I. Low and R. Vega-Morales, JHEP 1111, 027 (2011) [arXiv:1108.2274 [hep-ph]].

[99] R. Boughezal, T. J. LeCompte and F. Petriello, arXiv:1208.4311 [hep-ph]. 